\title{
Cognitive Radio for Smart Grid: Theory, Algorithms, and Security
}

\author{
Raghuram Ranganathan, ${ }^{1,2}$ Robert Qiu,, 2 Zhen Hu, ${ }^{1,2}$ Shujie Hou, ${ }^{1,2}$ \\ Marbin Pazos-Revilla, ${ }^{1,2}$ Gang Zheng, ${ }^{1,2}$ Zhe Chen,, ${ }^{1,2}$ and Nan Guo ${ }^{1,2}$ \\ ${ }^{1}$ Department of Electrical and Computer Engineering, Center for Manufacturing Research, \\ Tennessee Technological University, Cookeville, TN 38505, USA \\ ${ }^{2}$ Cognitive Radio Institute, Tennessee Technological University, Cookeville, TN 38505, USA
}

Correspondence should be addressed to Raghuram Ranganathan, raghu_ucf@yahoo.com

Received 9 February 2011; Accepted 24 March 2011

Academic Editor: Chi Zhou

Copyright ( 2011 Raghuram Ranganathan et al. This is an open access article distributed under the Creative Commons Attribution License, which permits unrestricted use, distribution, and reproduction in any medium, provided the original work is properly cited.

\begin{abstract}
Recently, cognitive radio and smart grid are two areas which have received considerable research impetus. Cognitive radios are intelligent software defined radios (SDRs) that efficiently utilize the unused regions of the spectrum, to achieve higher data rates. The smart grid is an automated electric power system that monitors and controls grid activities. In this paper, the novel concept of incorporating a cognitive radio network as the communications infrastructure for the smart grid is presented. A brief overview of the cognitive radio, IEEE 802.22 standard and smart grid, is provided. Experimental results obtained by using dimensionality reduction techniques such as principal component analysis (PCA), kernel PCA, and landmark maximum variance unfolding (LMVU) on Wi-Fi signal measurements are presented in a spectrum sensing context. Furthermore, compressed sensing algorithms such as Bayesian compressed sensing and the compressed sensing Kalman filter is employed for recovering the sparse smart meter transmissions. From the power system point of view, a supervised learning method called support vector machine (SVM) is used for the automated classification of power system disturbances. The impending problem of securing the smart grid is also addressed, in addition to the possibility of applying FPGA-based fuzzy logic intrusion detection for the smart grid.
\end{abstract}

\section{Introduction}

1.1. Cognitive Radio. Cognitive radio (CR) is an intelligent software defined radio (SDR) technology that facilitates efficient, reliable, and dynamic use of the underused radio spectrum by reconfiguring its operating parameters and functionalities in real time depending on the radio environment. Cognitive radio networks promise to resolve the bandwidth scarcity problem by allowing unlicensed devices to transmit in unused "spectrum holes" in licensed bands without causing harmful interference to authorized users [14]. In concept, the cognitive technology configures the radio for different combinations of protocol, operating frequency, and waveform. Current research on cognitive radio covers a wide range of areas; including spectrum sensing, channel estimation, spectrum sharing, and medium access control (MAC).

Due to its versatility, CR networks are expected to be increasingly deployed in both the commercial and military sectors for dynamic spectrum management. In order to develop a standard for CRs, the IEEE 802.22 working group was formed in November 2004 [5]. The corresponding IEEE 802.22 standard defines the physical (PHY) and medium access control (MAC) layers for a wireless regional area network (WRAN) that uses white spaces within the television bands between 54 and $862 \mathrm{MHz}$, especially within rural areas where usage may be lower. Details of the IEEE 802.22 standard including system topology, system capacity, and the projected coverage for the system are given in the next section.

1.2. The 802.22 System. The IEEE 802.22 is the first standardized air interface for CR networks based on opportunistic utilization of the TV broadcast spectrum $[6,7]$. The main objective of the IEEE 802.22 standard is to provide broadband connectivity to remote areas with comparable performance to broadband technologies such as cable and 
DSL, in urban areas. In this regard, the FCC selected the predominantly unoccupied TV station channels operating in the VHF and UHF region of the radio spectrum.

1.2.1. System Topology. The 802.22 system is a point-tomultipoint wireless air interface consisting of a base station (BS) that manages a cell comprised of number of users or customer premises equipments (CPEs) [8]. The BS controls the medium access and "cognitive functions" in its cell and transmits data to the CPEs in the downlink, while receiving data in the uplink direction from the CPEs. The various CPEs perform distributed sensing of the signal power in the various channels of the TV band. In this manner, the BS collects the different measurements from the CPEs and exploits the spatial diversity of the CPEs to make a decision if any portion of the spectrum is available.

1.2.2. Service Coverage. Compared to other IEEE 802 standards such as 802.11 , the 802.22 BS coverage range can reach up to $100 \mathrm{KM}$, if not limited by power constraints. The coverage of different wireless standards is shown in Figure 1. The WRAN has the highest coverage due to higher transmit power and long-range propagation characteristics of TV bands.

1.2.3. System Capacity. The WRAN systems can achieve comparable performance to that of DSL, with downlink speeds of $1.5 \mathrm{Mbps}$ and uplink speed of $384 \mathrm{Kbps}$. The system would thus be able to support 12 simultaneous CPEs, resulting in an overall system download capacity of $18 \mathrm{Mbps}$.

The specification parameters of the IEEE 802.22 standard is summarized in Table 1.

1.3. Smart Grid. Smart grid explores and exploits two-way communication technology, advanced sensing, metering and measurement technology, modern control theory, network grid technology, and machine learning in the power system to make the power network stable, secure, efficient, flexible, economical, and environmentally friendly.

Novel control technology, information technology, and management technology should be effectively integrated to realize the smart information exchange within the power system from power generation, power transmission, power transformation, power distribution, power scheduling to power utilization. The goal of smart grid is to systematically optimize the cycle of power generation and utilization.

Based on open-system architecture and shared information mode, power flow, information flow, and transaction flow can be synchronized. In this way, the operation performance of power enterprises can be increased. From power customer's perspective, demand response should be implemented. Customers would like to participate in more activities in the power system and power market to reduce their electric bills.

Distributed energy resources, for example, solar energy, wind energy, and so on, should also play an important role in the smart grid. Versatile distributed energy resources can perform the peak power shaving and increase the
TABLE 1: IEEE 802.22 characteristics.

\begin{tabular}{lc}
\hline Parameter & Specification \\
\hline Typical cell radius $(\mathrm{km})$ & $30-100 \mathrm{~km}$ \\
Methodology & Spectrum sensing to \\
Channel bandwidth $(\mathrm{MHz})$ & identify free channels \\
Modulation & OFDM \\
Channel capacity & $18 \mathrm{Mbps}$ \\
User capacity & Downlink: $1.5 \mathrm{Mbps}$ \\
& Uplink: $384 \mathrm{kbps}$ \\
\hline
\end{tabular}

stability of the power system. However, distributed energy generation imposes new challenges on the power system. Power system planning, power quality issue, and so on should be reconsidered.

To support the smart grid, a dedicated two-way communications infrastructure should be set up for the power system. In this way, secure, reliable, and efficient communication and information exchange can be guaranteed. In addition, the various devices, equipments, and power generation facilities of the current power system should be updated and renovated. Novel technologies for power electronics should be used to build advanced power devices, for example, transformer, relay, switch, storage, and so on.

To incorporate the smart features into the power system, computationally intelligent techniques, that is, machine learning and dimensionality reduction, should be widely applied. Machine learning is a scientific discipline that is concerned with the design of algorithms for computers to imitate the behavior of human beings, which includes learning to recognize complex patterns and make decisions based on experience, automatically and intelligently. Dimensionality reduction is the process of reducing the number of random variables under consideration to control the degrees of freedom. Several areas for applying computationally intelligent techniques to the smart grid have been identified in [9]. These areas are smart sensing and metering, autonomous control, adaptive protection, advanced data management and visualization, intelligent interfaces with distributed resources and market, decision support systems for system operation, and planning. The concept of compressing electrical power grids using the singular value decomposition (SVD) analysis is proposed in [10] to reduce the network traffic. The main idea is to determine what parts of the system are more strongly coupled from the grid admittance matrix [10].

In the smart grid, there will be more than one element, agent, controller, or decision maker. The control algorithm for the system with a single agent cannot be well suited for the distributed control or noncooperative control. From the multiagent control issue perspective, Game theory gives a general control methodology to deal with interaction, competition, and cooperation among decision makers in the complex system. Game theory is widely used in social sciences, economics, engineering, and so on. For the smart grid, the energy consumption scheduling issue has been 


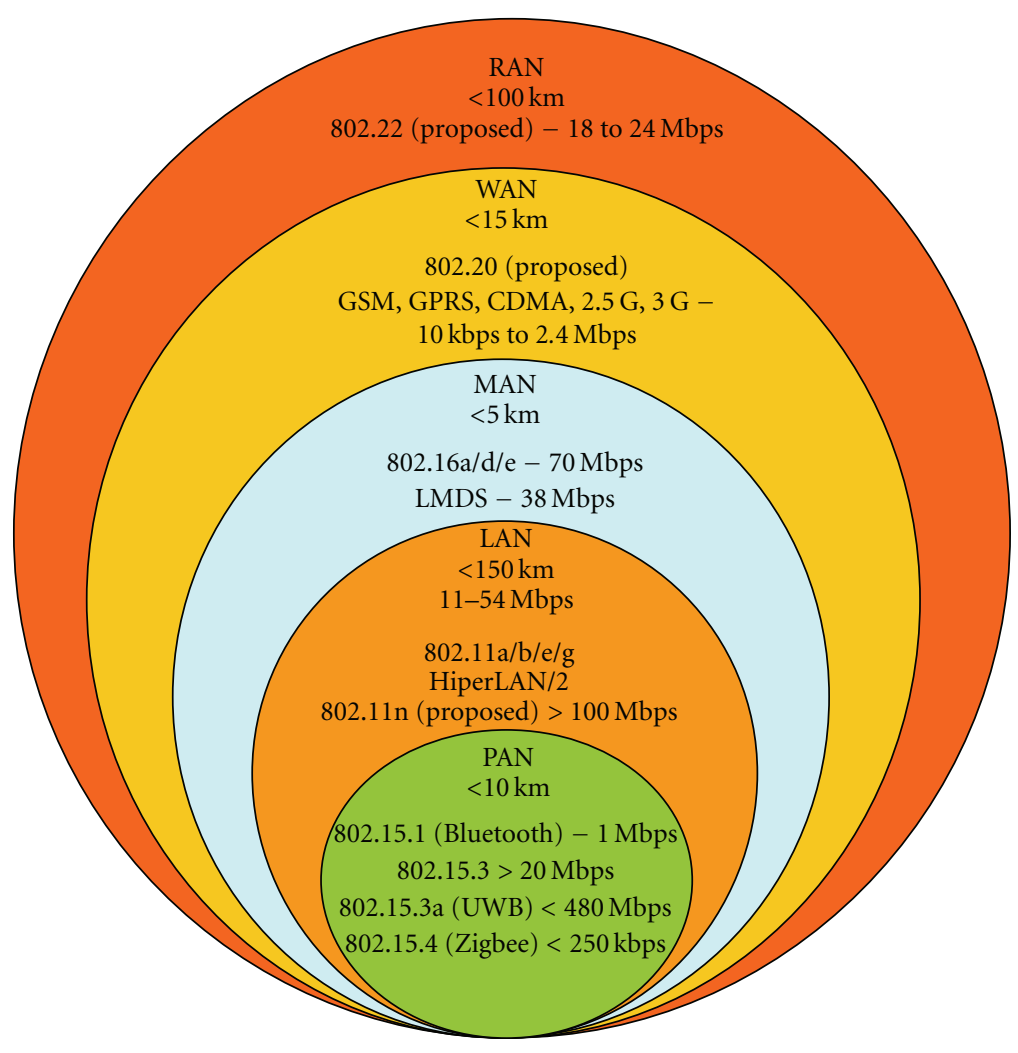

FIGURE 1: Comparison of 802.22 with other wireless standards.

formulated as a game theory problem [11]. The aim of scheduling is to reduce the total energy cost as well as PAR in the load demand [11]. Based on the assumption that the charge for each subscriber is proportional to his/her total daily load, the energy consumption game can be solved distributively with minimum exchanging information [11]. Meanwhile, the unique Nash equilibrium of the energy consumption game is the optimal solution to the central scheduling problem [11]. The work in [11] has been extended by [12]. Different control strategies based on the degree of information sharing in the network have been studied [12]. Partial knowledge setting and blind setting are considered. The proposed distributed stochastic energy consumption scheduling algorithms can still successfully exploit the limited information to improve the overall load profile [12]. In the context of the electrical power system, auction theory is a popular approach to deal with the power control issue from an economic point of view. Auction theory is a kind of game theory that deals with the behavior of agents in auction markets. Operation of a multiagent system for microgrid control has been presented in [13]. Auction theory has been exploited as the foundation of the proposed algorithm, the main idea being that every distributed energy resource or controllable load decides what is best for it, taking into account the overall benefit [13].

From the discussion of the control algorithm in smart grid, it is safe to say that pricing-based algorithms, pricingbased utility functions, and pricing models should be considered to optimize the system. A quasidynamic pricing model [14] has been proposed to minimize the electricity bill of cooperative users. The price comprises of a base price and a penalty term. Two methods are described. Deadlinedriven continuous variable method is suitable for an energy cost optimization with less interruptible tasks, while the time slot-based method is appropriate for more interruptible tasks [14]. One optimal real-time pricing algorithm has been mentioned in [15]. Energy pricing is an essential tool to develop efficient demand-side management strategies [15]. The proposed algorithm can be implemented in a distributed manner to maximize the aggregate utility of all users and minimize the cost imposed to the energy provider [15].

1.4. Power System Disturbance Classification. It is critical for power system operators to discern power system disturbances characteristics in order to take control measures to ensure power system security and reliability. This issue has become even more challenging due to the expansion of power system interconnections and integration of distributed generators and renewable energy resources $[16,17]$. Wide area measurement system (WAMS) implements the disturbance detection by collecting measurements from local sensors (wire, cable, or wireless) and managing it on a central server [18-22].

Power system disturbance classification has been conducted using pattern recognition in [23-25] based on power system measurements like voltage quality, power flow, or 
frequency. Artificial intelligence, such as neural networks, and particle swarm optimization have been introduced for complex signal classification $[26,27]$. However, the implementation of these algorithms is too complicated and not practical. To circumvent this, a machine-learning method called support vector machine (SVM) can be employed to quickly and accurately classify various common power system disturbances using the frequency data at various points in the power system. The objective is to eventually enable automated classification of disturbances, which is currently easy for a person to accomplish, yet difficult for a computer. SVM, which was first proposed by Cortes and Vapnik [28], transforms unknown data from a nonlinear space to a linear space. Subsequently, any linear algorithm can be applied to form the knowledge for the machine operation. SVM can achieve a unique global optimum for a convex optimization problem. In addition, SVM is not affected by the uncertainty in the parameters. Due to its various advantages, SVM is increasingly preferred in the field of pattern recognition and classification $[26,27]$.

1.5. Securing the Smart Grid. The smart grid is aimed at transforming the already aging electric power grid in the United States into a digitally advanced and decentralized infrastructure with heavy reliance on control, energy distribution, communication, and security.

In order to develop this infrastructure, a high level of interconnectivity and reliability among its nodes is required. Sensors, advanced metering devices, electrical appliances, and monitoring devices, just to mention a few, will be highly interconnected allowing for the seamless flow of data. Reliability and security in this flow of data between nodes, as shown in Figure 2, is crucial due to the low latency and cyber attacks resilience requirements of the smart grid.

A distributed interconnection among these nodes will be ubiquitous, just as finding a similar level of connectivity among cellular phones or computing nodes in a large organization. The smart grid environment, however, poses a new set of communications and security paradigms. Due to their complexity and importance to the realization of the smart grid infrastructure, it is extremely important to study the interactions among the nodes, more specifically, in terms of their communications and security.

Taking into account that reliability and security will impose constraints on the majority of the devices connected to the Smart Grid, if not all, it would be wise to consider communication standards, protocols, and devices that are designed from the ground up to be secured, logically and physically. Since a great portion of the traffic generated within the grid will be traveling on an unsecured medium such as the Internet, it is imperative to minimize the amount of potential security loopholes. Additionally, the human variable should also be taken into account in the security model, as part of the security infrastructure.

When it comes to security, communication is key, and information should be properly disseminated to all the parties involved, ensuring that everyone has a clear and common understanding of security needs facilitating their implementation and operation. Training and informing users about processes, study of human behavior, and the perception of events related to the processes is as important to the entire security equation, as it is to engineer a secured infrastructure. As a matter of fact, the greatest security threat to any infrastructure is human error, as opposed to the technology securing it. Communications in the smart grid is a key component of the entire infrastructure, and logically we divide it into two sections, the backbone communications (interdomain), which will carry communications among domains such as those shown in Figure 2, and the communications at the local area network (intradomain) limited by perimeters such as a customer's house, or a distribution facility [29]. It would be important to note that due to current limitations, the focus of research on our testbed will be on intradomain communications, without disregard for future considerations of the interdomain aspect.

We can say that current and emerging technologies in telecommunications, most of which are expected to fall in the wireless realm (Wimax, Zigbee, 802.11, etc.), can accommodate the communications needs of both inter and intradomain environments, however, not without flaws. From a security standpoint, these technologies are not designed to be secure from the ground up. For example, Zigbee is a standard for short-range communications, and manufacturers of Zigbee compliant chips produce them without necessarily considering the security issue. In addition, chip manufacturers print the chip model on top of the chip itself as a standard practice. The chip specifications can therefore be easily downloaded, and potential flaws of the chip can be easily exploited by attackers. Additionally, by default, many of these chips do not carry any internal security features and, therefore, rely on external chips, or on higher level software applications for this purpose. An easy access to the external chip by any malicious attacker could potentially disable any installed security features. This and other similar scenarios lead us to think that the smart grid should be driven by technologies and standards that consider security as their primary concern.

The smart grid has been conceived as being distributed in nature and heavily dependent on wireless communications. Today's SOHO (small office/home office) and enterprisegraded wireless devices include security features to mitigate attacks, the vast majority still relying on conventional rulebased detection. It has been shown that conventional rulebased detection systems, although helpful, do not have the capability of detecting unknown attacks. Furthermore, as presented in [30], these conventional IDSs would not be able to detect such an attack if it is carefully crafted, since the majority of these rules are solely based on strict thresholds.

\section{Review}

2.1. Cognitive Radio Network Testbed. A Cognitive Radio (CR) network testbed is being built at Tennessee Technological University $[31,32]$. The idea of applying a cognitive radio network testbed to the smart grid was developed at Tennessee Technological University in the middle of 2009 in a funded research proposal [33]. Subsequently, this idea has been strengthened in [31, 34-37]. The objective of this 


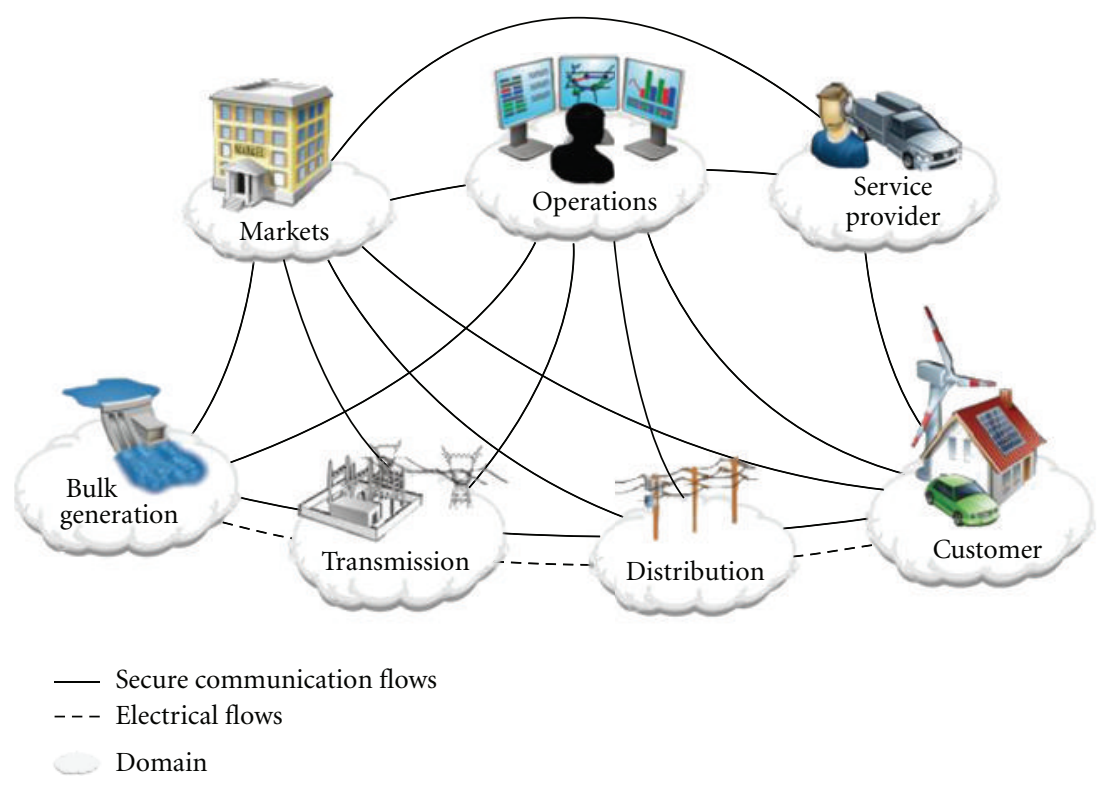

FIGURE 2: Interaction among actors in smart grid domains through secure communication flows and flows of electricity.

testbed is to achieve the convergence of cognitive radio, cognitive radar, and smart grid [38].

The cognitive radio network testbed being built is unique and real-time oriented. It is designed to provide much more stand-alone computing power and reduce the response time delay. The cognitive radio network testbed is comprised of tens of nodes, with each node based on a self-designed motherboard, and commercial radio frequency (RF) boards. On the self-designed motherboard, there are two advanced and powerful field programmable gate arrays (FPGAs) that can be flexibly configured to implement any function. Therefore, this network testbed can also be applied to the smart grid.

To our understanding, the benefits of applying cognitive radio to the smart grid are summarized in Table 2. Firstly, cognitive radio can operate over a wide range of frequency bands. It has frequency agility. This feature is especially useful for smart grid because the frequency spectrum today is so crowded, and cognitive radio provides the capability of reusing unused frequency bands for the smart grid. Secondly, cognitive radio enables high-speed data transmission for the smart grid. This is due to the wide-band nature of cognitive radio. The data rate can be as high as tens of Mbps, in contrast to the ZigBee that can only provide a data rate of tens to hundreds of kbps. Thirdly, cognitive radio has the potential to transmit data over a long-distance. Recently, the federal communications commission (FCC) has decided to allow using unused TV bands for wireless communications. The TV bands are ideal for long distance mass data transmission. Cognitive radio in a wireless regional area network (WRAN) scenario is designed to utilize the unused TV bands. Employing cognitive radio, the smart grid can communicate over a long distance over the air. Fourthly, cognitive radio boasts of cognitive learning and adaptation capability. It has the ability to learn the environment, reason
TABLE 2: Advantages of applying cognitive radio to smart grid.

\begin{tabular}{ll}
\hline Salient features & \multicolumn{1}{c}{ Description } \\
\hline Frequency diversity & $\begin{array}{l}\text { CR can operate over unused frequency } \\
\text { bands } \\
\text { Transmission speed } \\
\text { Data rates of up to tens of Mbps can be } \\
\text { achieved } \\
\text { CR can transmit over long distances in a } \\
\text { WRAN scenario }\end{array}$ \\
Adaptability & $\begin{array}{l}\text { CR has inherent intelligence to adapt to } \\
\text { changes in the environment } \\
\text { Built on an SDR platform, the CR can be } \\
\text { selectively programmed }\end{array}$ \\
\hline
\end{tabular}

from it, and adapt accordingly. Cognitive radio makes the smart grid "smarter" and more robust. Fifthly, cognitive radio is based on the software defined radio (SDR) platform, which is a programmable radio. Hence, cognitive radio is capable of performing different applications and tasks. In addition, security, robustness, reliability, scalability, and sustainability of the smart grid can be effectively supported by cognitive radio due to its flexibility and reprogrammability.

2.2. Smart Grid Communications. Smart grid technology has attracted significant research focus in recent years from the power and communications standpoint $[39,40]$. CRs provide a promising solution to the growing spectrum scarcity problem by intelligently accessing unused regions of spectrum originally licensed to primary users (PUs). One of the key requirements for the smart grid is a robust and efficient communications infrastructure that can address both the current and future energy management needs $[41,42]$. With the advent of modern communications 
technologies, and the recently defined IEEE 802.22 standard, CR networks are believed to be a viable choice for smart grid applications. The opportunistic access of the TV broadcast spectrum as outlined in the 802.22 standard can be realized as one of the cognitive network functions.

\section{Examples}

In this section, examples are presented employing machine learning and signal processing techniques for dimensionality reduction, recovery of smart meter transmissions, power system disturbances classification, and fuzzy logic-based intrusion detection. This section is divided as follows. In Section 3.1, dimensionality reduction techniques such as PCA, KPCA, and LVMU, in combination with SVM, are used as a preprocessing tool in a spectrum sensing application for Wi-Fi signals. The SVM technique is used for power system disturbances classification in Section 3.2. In Section 3.3, the sparsity of the smart meter transmissions is exploited to recover the BPSK-modulated smart meter data, by employing the recently proposed bayesian compressed sensing, and compressed sensing kalman filter methods. Finally, the critical issue of smart grid security is addressed in Section 3.4, and a possible approach to realize this is provided using FPGA-based fuzzy logic.

\subsection{Dimensionality Reduction Applied to Cognitive Radio} with Experimental Validation. In radar and sensing signal processing, the control of degrees of freedom (DOF)or intrinsic dimensionality-is the first step, called preprocessing. The network dimensionality, on the other hand, has received attention in information theory literature. The techniques of the dimensionality reduction can be explored to extract the intrinsic dimensionality of the highdimensional data.

Dimensionality reduction methods are innovative and important tools in machine learning [43]. The original dimensionality data collected from our living world may contain a lot of features however, usually these features are highly correlated and redundant with noise. Hence, the intrinsic dimensionality of the collected data is much fewer than the original features. Dimensionality reduction attempts to select or extract a lower dimensionality expression but retain most of the useful information. In the first example, both linear methods such as principal component analysis (PCA) [44], nonlinear methods such as kernel principal component analysis (KPCA) [45], and landmark maximum variance unfolding (LMVU) $[46,47]$ are studied, by combining them with the support vector machine (SVM) [48-53] — the latest breakthrough in machine learning, in the context of spectrum sensing for cognitive radio.

Measured Wi-Fi signals with high signal-to-noise ratio (SNR) are employed in the first example. The DOF of the Wi-Fi signals is extracted by three dimensionality reduction techniques in this example. The advantages of applying dimensionality reduction techniques are verified by comparing with the results obtained without dimensionality reduction.
3.1.1. Wi-Fi Signal Measurements. Wi-Fi time-domain signals have been measured and recorded using an advanced digital phosphor oscilloscope (DPO) whose model is Tektronix DPO72004 [54]. The DPO supports a maximum bandwidth of $20 \mathrm{GHz}$ and a maximum sampling rate of $50 \mathrm{GS} / \mathrm{s}$. It is capable of recording up to $250 \mathrm{M}$ samples per channel. In the measurements, a laptop accesses the Internet through a wireless Wi-Fi router, as shown in Figure 3. An antenna with a frequency range of $800 \mathrm{MHz}$ to $2500 \mathrm{MHz}$ is placed near the laptop and connected to the DPO. The sampling rate of the DPO is set to $6.25 \mathrm{GS} / \mathrm{s}$. Recorded timedomain Wi-Fi signals are shown in Figure 4. The duration of the recorded Wi-Fi signals is $40 \mathrm{~ms}$.

The recorded $40 \mathrm{~ms}$ Wi-Fi signals are divided into 8000 slots, with each slot lasting $5 \mu$ s. These slots can be viewed as spectrum sensing slots. The time-domain Wi-Fi signals within the first $1 \mu \mathrm{s}$ of every slot are then transformed into the frequency domain using the Fast Fourier Transform (FFT), which is equivalent to FFT-based spectrum sensing. In this paper, the frequency band of $2.411-2.433 \mathrm{GHz}$ is considered. The resolution in the frequency domain is $1 \mathrm{MHz}$. Therefore, for each slot, 23 points in the frequency domain can be obtained, of which 13 points will be selected in the following experiment.

3.1.2. Experimental Validation. SVM will be exploited to classify the states (busy $l_{i}=1$ or idle $l_{i}=0$ ) of the measured $\mathrm{Wi}-\mathrm{Fi}$ data with or without dimensionality reduction, given the true states. SVM will classify the states of the spectrum data at different time slots.

The DOF of the Wi-Fi frequency domain signals is extracted using the original 13 dimensions. The flow chart of the SVM processing combined with dimensionality reduction methods, including data processing, is shown in Figure 5.

The false alarm rate obtained by combining SVM with dimensionality reduction and employing only SVM is shown in Figure 6. The results are averaged over 50 experiments. In each experiment, the number of training sets is 200 , and the number of testing sets is 1800 .

The original dimension of the frequency domain data is varied from 1 to 13 for the SVM method. In addition, the SVM method is combined with the extracted dimensions from 1 to 13 , obtained with dimensionality reduction.

In the whole experiment, a Gaussian RBF kernel with $2 \sigma^{2}=5.5^{2}$ is used for KPCA. The parameter $k=3$, in which $k$ is the number of nearest neighbors of $y_{i}\left(x_{i}\right)$ (including both training and testing sets) for LMVU. The optimization toolbox SeDuMi 1.1R3 [55] is applied to solve the optimization step in LMVU. The SVM toolbox SVMKM [56] is used to train and test the SVM processes. The kernels selected for SVM are heavy-tailed RBF kernels with parameters $\gamma=1, a=1$, and $b=1$. These parameters are kept constant for the whole experiment.

Experimental results show that with dimensionality reduction, the spectrum sensing performance is much better with fewer features than that without dimensionality reduction. 


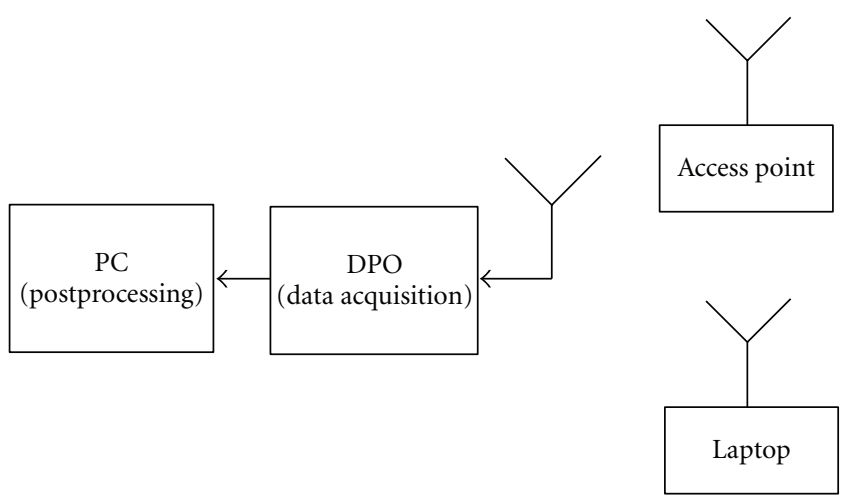

FIGURE 3: Setup of the measurement of Wi-Fi signals.

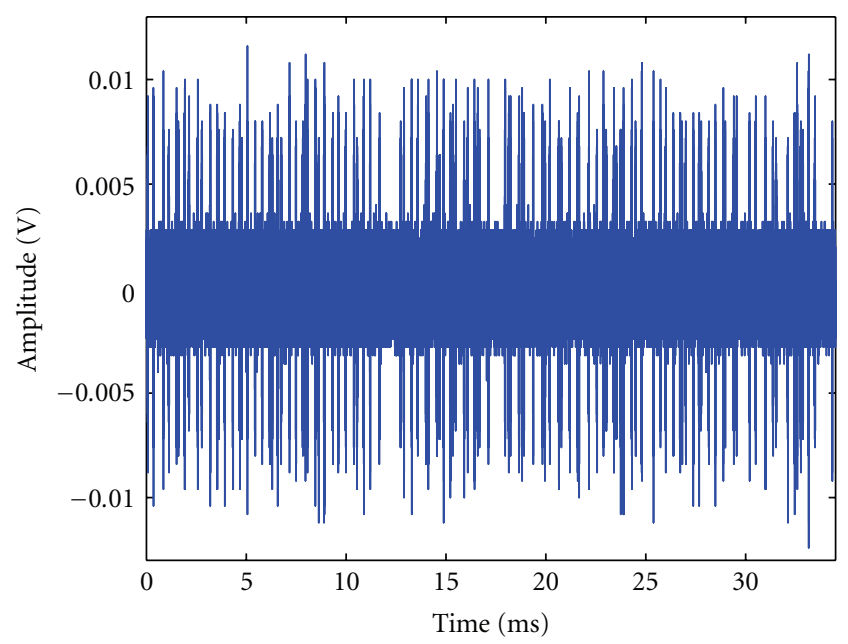

FIgURE 4: Recorded Wi-Fi signals in time domain.

3.2. Classifying Power System Disturbances Using SVM. Due to the large variability exhibited by the few power system disturbance data sets available, data sets are generated mathematically in this section for training purposes. In this way, a large number of data sets can be generated and used in training to ensure accurate SVM models.

Based on frequency and time domain analysis, and derived from typical disturbances, mathematical models for each disturbance can be formulated by the following signal equations:

(i) generation trip,

$$
\begin{array}{r}
u(t)=\sin \left(\omega_{0} t\right)+\alpha_{1} e^{-c_{1}\left(t_{11}-t_{12}\right)} \sin \left(\beta_{1} \omega_{0}\left(t_{11}-t_{12}\right)\right)+\cdots \\
\alpha_{2} e^{-c_{2}\left(t_{21}-t_{22}\right)} \sin \left(\beta_{2} \omega_{0}\left(t_{11}-t_{12}\right)\right)+\cdots
\end{array}
$$

(ii) line trip,

$$
u(t)=\left(1-\alpha\left(u\left(t_{2}\right)-u\left(t_{1}\right)\right)\right) \sin \left(\omega_{0} t\right),
$$

(iii) frequency oscillation,

$$
u(t)=\sin \left(\omega_{0} t\right)+\alpha \mathrm{e}^{-c\left(t-t_{1}\right)} \sin \left(\beta \omega_{0}\left(t-t_{1}\right)\right) \prod\left(t_{2}-t_{1}\right),
$$

where various terms are defined as follows: time window $\prod\left(t_{2}-t_{1}\right)=u\left(t_{2}\right)-u\left(t_{1}\right), \omega_{0}$ is a random frequency of basic frequency fluctuation, $\alpha$ is an additional signal factor, $\beta$ is an additional frequency factor, $c$ is the time constant, and $t$ is the time period.

After carefully selecting the obtained parameters, such as amplitude, angles, frequency, and time period of each subfrequency component, enough examples can be generated by manipulating these parameters by random deviation within a tolerance range. Figures 7, 8, and 9 are the mesh patterns of 200 mathematical examples for the generation trip, line trip, and frequency oscillations, respectively.

SVM is a linear classifier in the parameter space, but it becomes a nonlinear classifier as a result of the nonlinear mapping of the space of the input patterns into the highdimensional feature space [27]. Training an SVM model is a quadratic-optimization problem $[57,58]$. The hyperplane represented by $\langle\omega, x\rangle+b=0$ is constructed, so that the margin between the hyperplane and nearest point is maximized, where $\omega$ is the vector of hyperplane coefficients, $b$ is a bias term, and $\langle\cdot\rangle$ denotes the inner product of two vectors.

Therefore, the classification function is $f(x ; \omega, b)=$ $\langle\omega, x\rangle+b$. An $n$-class classifier is constructed using the maximum value of the function $f_{i j}(x ; \omega, b)=\left\langle\omega_{i j}, x\right\rangle+b_{i j}$, $k=1, \ldots, n$. For SVM, the problem can be solved by training data $x_{i}^{k}$, where $i=1, \ldots, m$ data points.

Thus, the mathematical function between class $i$ and class $j$ is represented by the following equations:

$$
\begin{aligned}
& f_{i j}(x ; \omega, b)=\left\langle\omega_{i j}, x\right\rangle+b_{i j} \\
& \text { Minimize: } \frac{1}{2}\left\langle\omega_{i j}, \omega_{i j}^{T}\right\rangle+\frac{C}{n} \sum_{n=1}^{N} \xi_{n}^{i j} \\
& \text { Constraints: } y_{n}^{i j}\left(\left\langle\omega_{i j}, \omega_{i j}^{T}\right\rangle+b_{i j}\right) \geq 1-\xi_{n}^{i j},
\end{aligned}
$$

where $\xi_{n}^{i j} \geq 0$,

$$
y_{n}^{i j}= \begin{cases}+1 & \text { if } y_{n}=i \text { th class } \\ -1 & \text { if } y_{n}=j \text { th class }\end{cases}
$$

The machine-learning package Weka is used to classify the data using an SVM multiclass algorithm. After being transformed to the Weka format, the input data includes three categories: generation trip, oscillation, and line trip. The data contains 200 examples of each category which is then divided into 2 equal sized groups. The first set is used for training, and the second set is used for verification. The verification results are as follows: generation trip can be classified with a success rate of 0.985 , compared to 0.853 for the line trip, and 0.626 for the frequency oscillation.

3.3. Compressed Sensing-Based Smart Meter Reading. Compressed sensing, also known as compressive sensing, compressive sampling, or sparse sampling, is a technique for finding sparse solutions to underdetermined linear systems [59-63]. The concept of applying compressed sensing to smart meter reading was first proposed in [64]. A smart 


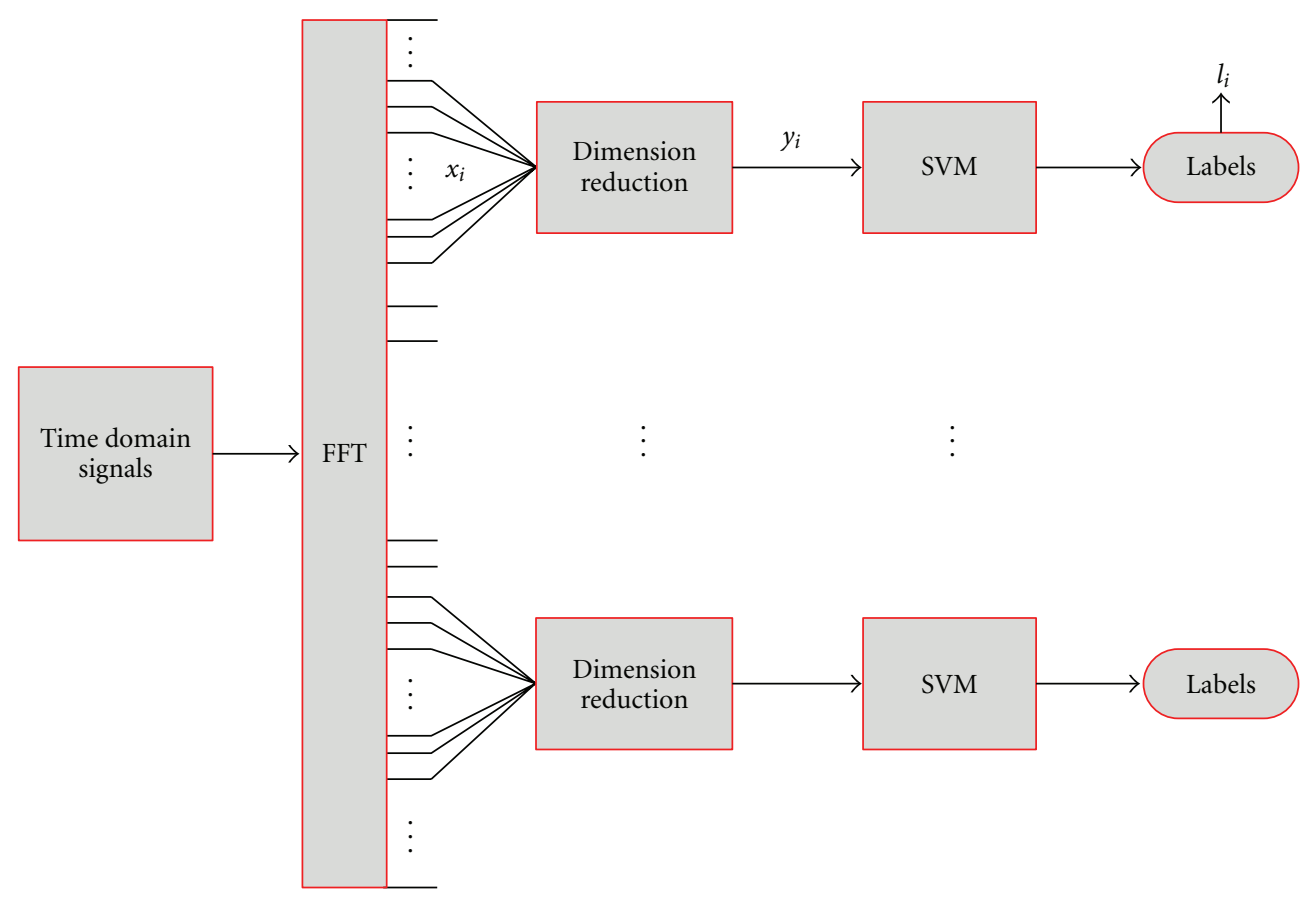

FIGURE 5: The flow chart of SVM combined with dimensionality reduction.

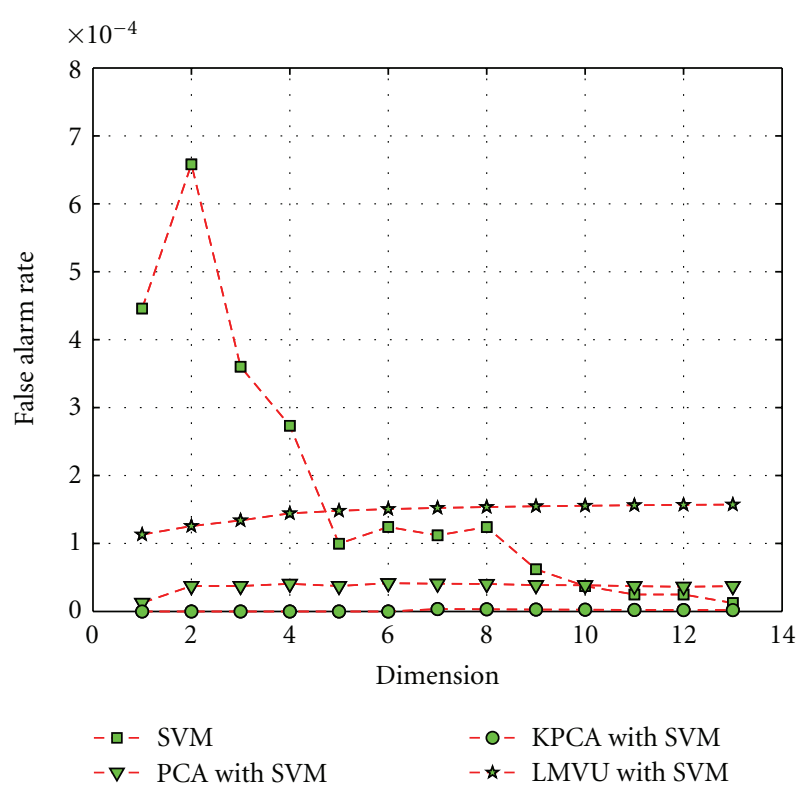

Figure 6: False alarm rate.

meter is an intelligent electrical meter that conveys information to the central power station regarding significant changes in the power load either through two-way wireless or power line communications. Since the power consumption in a particular home does not dynamically vary, the number of smart meters simultaneously transmitting is very small compared to the total number of meters in a particular cognitive smart grid network. As a result, the sparsity of the smart meter data transmission to the central processing node

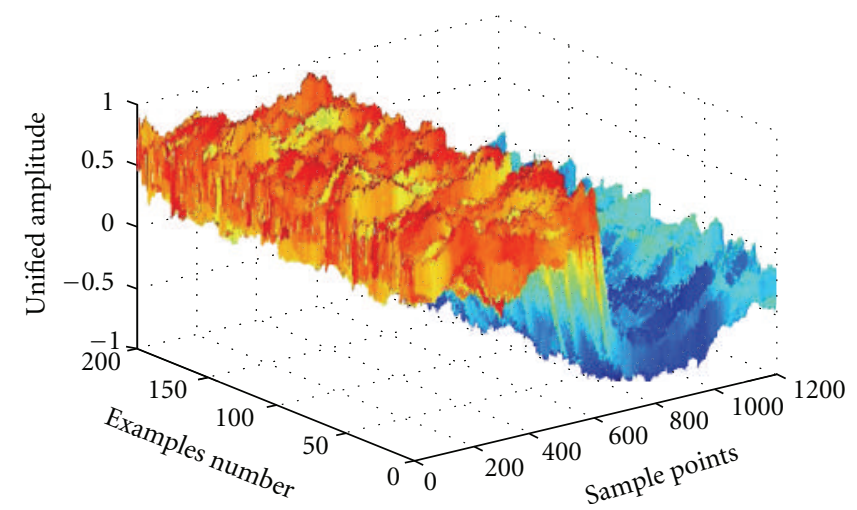

Figure 7: Mesh patterns of 200 examples for generation trip.

or access point (AP) was exploited in [64] for applying the principle of compressed sensing. However, in [64], it was assumed that the noise is bounded. In this paper, the newly proposed techniques of Bayesian compressive sensing [65], and compressed sensing kalman filter [66] are applied for smart meter reading when the noise is Gaussian distributed.

3.3.1. Bayesian Compressed Sensing. Consider an N-dimensional signal $y$ that is compressible in some basis function $A$, that is, $y$ can be accurately reconstructed with a small number $K$ of basis-function coefficients, where $K \ll N$. In other words, the basis coefficient vector $s$ is a sparse vector with a majority of components close to 0 . Compressive sensing states that it is possible to recover these basisfunction coefficients with fewer measurements $M<N$ of $y$. This is accomplished by a linear transformation of $y$ 


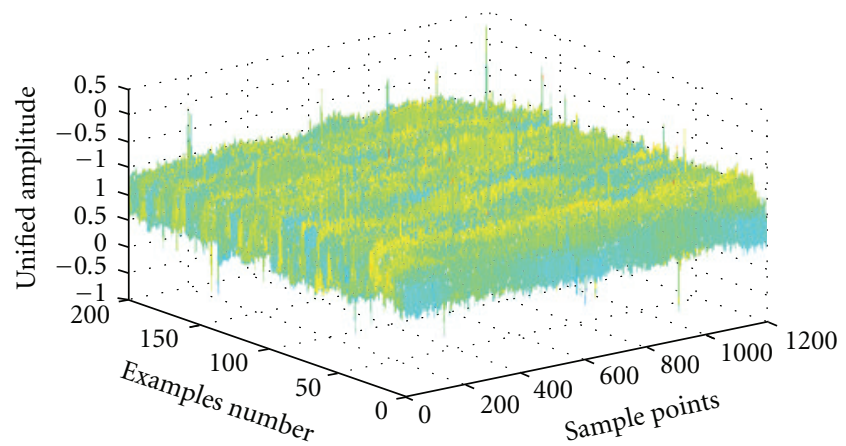

FIGURE 8: Mesh patterns of 200 examples for line trip.

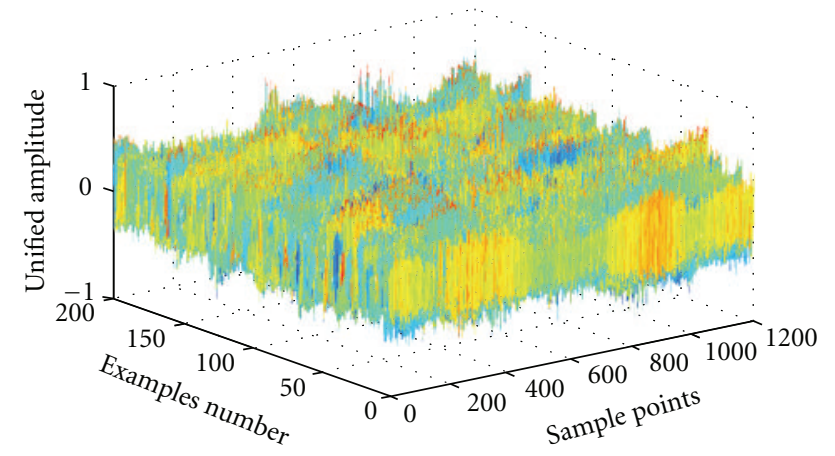

FIGURE 9: Mesh patterns of 200 examples for frequency oscillation.

onto an $M \times N$ matrix $H$ to generate an $M$-dimensional measurement vector $z$. Mathematically, $z$ can be represented as

$$
z=y^{T} A H
$$

Since we have $y=A s,(5)$ becomes

$$
z=H s \text {. }
$$

The expression in (6) is an underdetermined system; hence, the estimate of $s$ is ill conditioned. However, since $s$ is sparse with respect to $A,(6)$ can be solved as a $l_{1}$ norm minimization problem as follows:

$$
\widehat{s}=\arg \min _{s}\left[\|z-H s\|_{2}^{2}+\lambda\|s\|_{1}\right] .
$$

The scalar $\lambda$ decides the weightage given to the Euclidean error and the sparseness constraints in the first and second terms of (7), respectively.

The above optimization problem can be solved using many linear programming techniques such as basis pursuit (BP) [67], matching pursuit (MP) [68], and orthogonal matching pursuit (OMP) [69]. In [65], a Bayesian approach is employed to estimate $s$ from the compressed measurements $z$. Hence, by imposing a Laplace sparseness prior on $s$, and assuming a Gaussian likelihood model for $z$, the solution for (7) becomes a maximum a posterior (MAP) estimate for $s$.

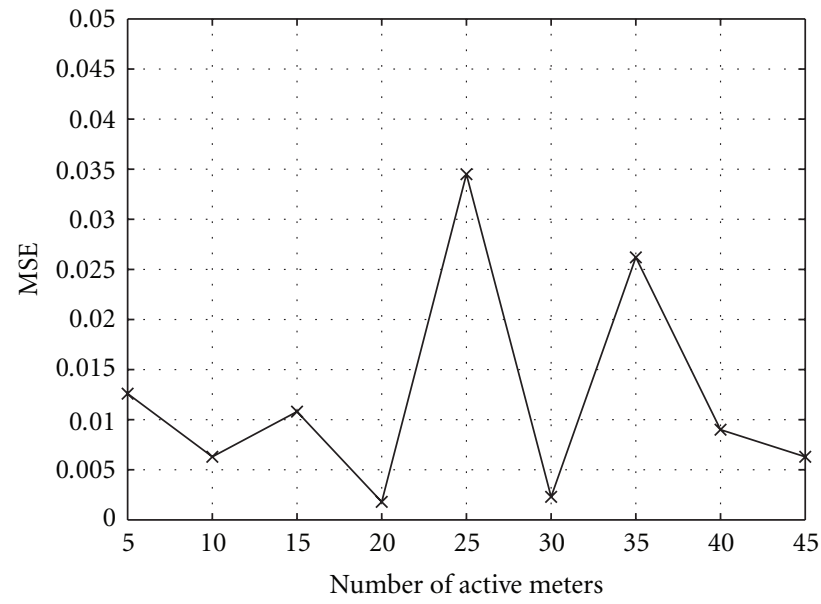

Figure 10: MSE achieved by BCS for different number of active meters.

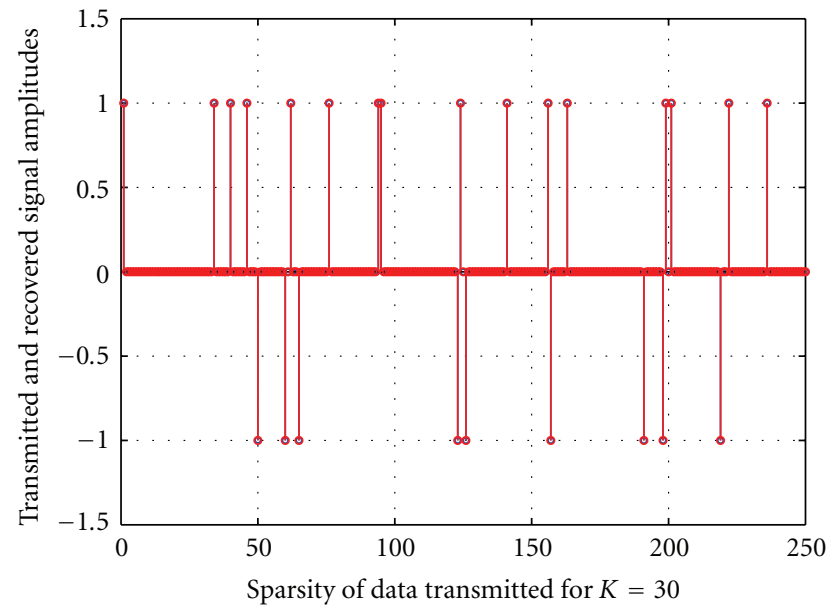

FIgURE 11: Sparsity of data transmitted for $K=30$.

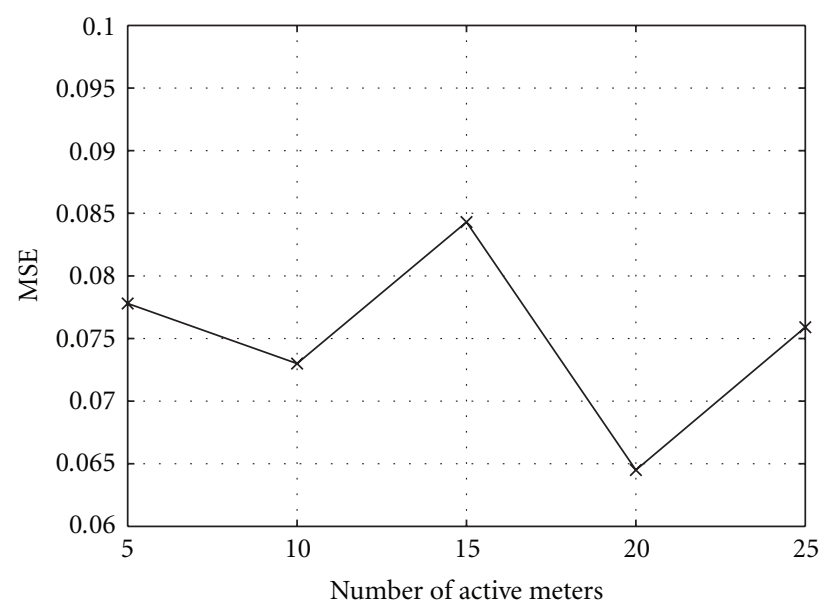

FIGURE 12: MSE achieved by CSKF for different number of active meters. 


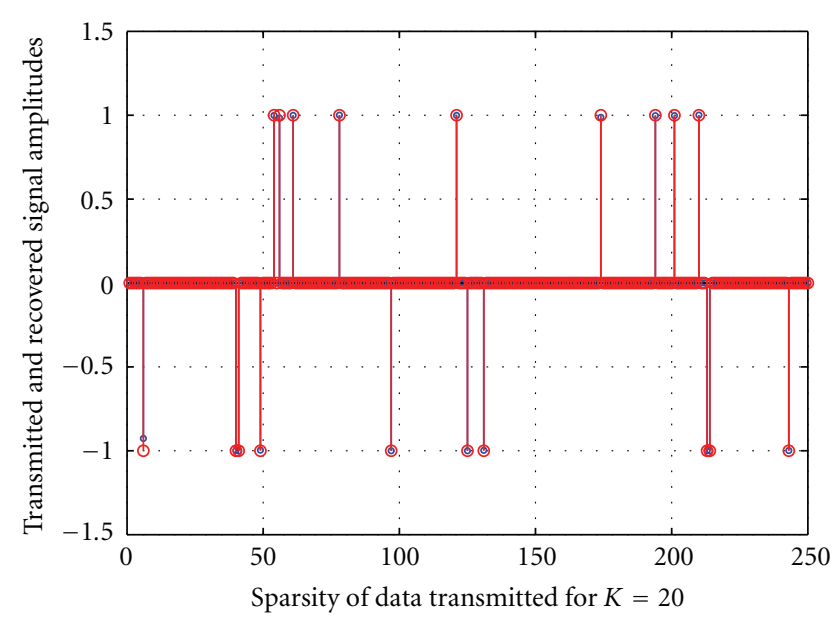

Figure 13: Sparsity of data transmitted for $K=20$.

3.3.2. Kalman Filter-Based Compressed Sensing. The kalman filter is a recursive filter that estimates the state of a process or system from a series of noisy observations [7072]. The kalman filter optimally estimates the true state of the system by making a prediction, estimating the error in the prediction, and computing a weighted average of the predicted value and the measured value. Therefore, the mathematical equations for the kalman filter can be separated into time updates or prediction and measurement updates or correction. Due to its versatility, the kalman filter has been used in diverse engineering applications such as digital signal processing, control systems, wireless communications, image processing, and weather forecasting [73-78].

Recently, a new kalman filtering approach for the recovery of sparse signals was proposed, called compressed sensing embedded kalman filter (CSKF-1) $[66,79]$. The CSKF1 adopts the pseudomeasurement (PM) technique [80] to incorporate a fictitious measurement in the kalman filtering process to satisfy the sparseness constraint. The PM can be expressed as

$$
\begin{gathered}
0=\hat{H} s-\delta, \\
\hat{H}=[\operatorname{sign}(s(1), s(2), \ldots, s(N))],
\end{gathered}
$$

where $\operatorname{sign}(s(k))=1$ if $s(k) \geq 0$, and -1 otherwise.

$\delta$ is the tuning parameter which controls the sparsity of the solution state vector.

3.3.3. System Description and Signal Model. As mentioned in the previous section, the smart meter data transmission to the AP is sparse in nature, that is, only a small percentage of meters would be actively transmitting data at any time. Therefore, the principles of compressed sensing can be readily applied to the recovery of the data reports. The main advantage of employing compressed sensing is that it allows the smart meters to transmit simultaneously, as opposed to the popular carrier sense multiple access (CSMA) protocol, which uses a random backoff to avoid collisions in transmissions. This could result in significant delay in data recovery.

The system consists of $N$ smart meters managed by an AP. In each frame, synchronization and channel estimation is performed, followed by data transmission. The synchronization and channel estimation can be performed by transmitting a pilot signal to the meters during the assigned periods in the frame and is beyond the scope of this paper. However, it is assumed that the channel parameters are flat fading in nature, with a large coherence time indicating a slow time-varying channel. The data transmission section in the frame is divided into several time slots during which the active smart meters can simultaneously transmit their readings. In mathematical form, the data transmission received by the AP at time $t$ can be expressed as

$$
z(t)=\sum_{i=1}^{N} p_{i}(t) c_{i} s_{i}+w(t)
$$

$c_{i}$ is the flat-fading channel parameter between meter $i$ and the AP, $p_{i}(t)$ is the pseudorandom spreading code at time $t$ for meter $i, s_{i}$ is the data transmitted by meter $i, w(t)$ is the Gaussian distributed noise term. The spreading code is known only to the AP and meters, preventing unauthorized people from accessing and tampering with the data. Suppose that the data transmission period has $T$ time slots, then the above signal model can be rewritten in matrix-vector form as follows:

$$
Z=H S+W
$$

where

$$
\begin{gathered}
H=P C, \\
C=\operatorname{diag}\left(c_{1}, c_{2}, \ldots, c_{N}\right), \\
P_{t, i}=\left[p_{i}(1), p_{i}(2), \ldots, p_{i}(T)\right]^{T}, \\
Z=[z(1), z(2), \ldots, z(T)]^{T}, \\
S=\left[s_{1}, s_{2}, \ldots, s_{N}\right]^{T}, \\
W=[w(1), w(2), \ldots, w(T)]^{T} .
\end{gathered}
$$

Since the number $K$ of meters simultaneously transmitting is small, $K \ll N$. As a result, the vector $S$ in (15) is sparse. It can therefore be easily inferred that (10) is identical to the CS equation (6), with the exception of additive Gaussian noise. Hence, the recovery of the smart meter reading would correspond to the optimization problem in (7). In the simulations, the total number of smart meters, $N$, in the cognitive smart grid network is considered to be 250, and the length of the observation vector/time slots $T$ is 100 . Binary phase shift keying (BPSK) modulation is considered. The mean square error (MSE) between the actual data vector and the estimated data vector is used as the measure of performance. The MSE is plotted for different number of active meters $K$ or sparsity of the data transmission. 


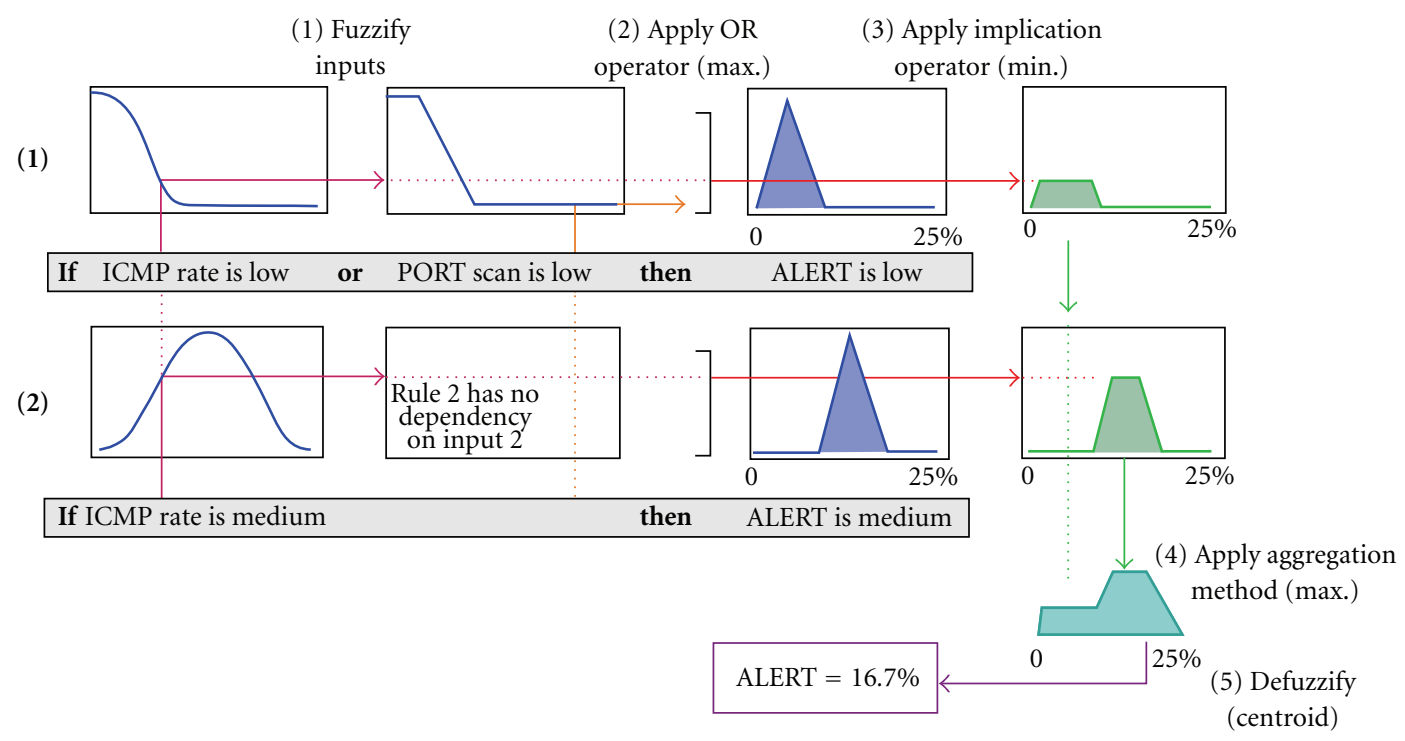

Figure 14: Fuzzy Logic example applied to IDS.

3.3.4. Bayesian Compressive Sensing for Smart Meter Reading. In this section, the smart grid data recovery problem in (6) is treated as a linear regression problem and is considered from a Bayesian perspective, as proposed in [65]. A hierarchical sparseness prior is imposed on $S$, and sparse Bayesian learning using the relevance vector machine (RVM) [81] is performed. The MSE achieved by the BCS algorithm for different values of $K$ is illustrated in Figure 10. For the case of $K=30$, the sparsity of the data transmitted along with the recovered data is illustrated in Figure 11.

3.3.5. Compressed Sensing Kalman Filter Approach. In this section, the recently proposed CSKF algorithm is employed for the recovery of data at the AP. The total number of smart meters in the network is assumed to be 250, and $T$ is 150 . The MSE yielded for various number of active meters $K$ is shown in Figure 12, respectively. A comparison between the amplitudes of the transmitted and recovered signal versus the sparsity of meter transmission is shown in Figure 13.

3.4. FPGA-Based Fuzzy Logic Intrusion Detection for Smart Grid. Artificial intelligence techniques such as fuzzy logic, bayesian inference, neural networks, and other methods can be employed to enhance the security gaps in conventional IDSs. As shown in Figure 14, a fuzzy logic approach was used in [82], in which different variables that influence the inference of an attack can be analyzed and later combined for the decision-making process of a security device. Additionally, if each security device serving as an IDS is aware not only of itself, but also of a limited number (depending on local resources and traffic) of surrounding trusted IDS devices, the alerts that these other devices generate can be used to adjust local variables or parameters to better cope with distributed attacks and more accurately detect their presence.

The research and development of robust and secure communication protocols, dynamic spectrum sensing, and distributed and collaborative security should be considered as an inherent part of smart grid architecture. An advanced decentralized and secure infrastructure needs to be developed with two-way capabilities for communicating information and controlling equipment, among other tasks, as indicated in the recently published "Guidelines for Smart Grid Cyber Security Vol.1” by the National Institute of Standards and Technologies. The complexity of such an endeavor, coupled with the amalgam of technologies and standards that will coexist in the development of the smart grid, makes it extremely necessary to have a common platform of development, with flexibility and reliable performance.

Field programmable gate arrays (FPGAs) development platforms share these advantages, not to mention the fact that a single silicon FPGA chip can be used to study several smart grid technologies and their implementations. FPGA chips offer significant potential for application in the smart grid for performing encryption and decryption, intrusion detection, low-latency routing, data acquisition and signal processing, parallelism, configurability of hardware devices, and high-performance and high-bandwidth tamper-resistant applications. Dr. William Sanders, a member of the Smart Grid Advisory Committee of the National Institute of Standards and Technology (NIST), has been in the recent years among the most influential persons in the research of smart grid security. His research team and several collaborating universities proposed the use of a Trustworthy Cyber Infrastructure for the Power Grid (TCIPG) that focuses on the security of low-level devices and communications, as well as trustworthy operation of the power grid under a variety of conditions including cyber attacks and emergencies [83]. TCIPG proposes a coordinated response and detection at multiple layers of the cyber-infrastructure hierarchy including but not limited to sensor/actuator and substation levels. At these levels of the hierarchy, software defined radio and wireless communications technologies could be used and studied to prevent attacks such as wireless jamming. 
Dr. Sanders also proposes the use of specifications-based IDS in protecting advanced metering infrastructures (AMIs) [84]. A distributed FPGA-based network with adaptive and cooperative capabilities can be used to study several security and communication aspects of this infrastructure both from the attackers and defensive point of view.

\section{Conclusions}

In this paper, the integration of two emerging technologies, namely, the cognitive radio and smart grid is addressed. The concept of dimensionality reduction is presented as a possible preprocessing method to extract the intrinsic dimensionality of high-dimensional data. Using Wi-Fi signal measurements, the effectiveness of the PCA, KPCA, and LVMU dimensionality reduction techniques in conjunction with the SVM method is provided in a spectrum sensing application. In addition, the SVM technique is used for suitably classifying the power system disturbances. For the recovery of sparse smart meter transmissions, experimental results obtained by employing the Bayesian compressed sensing and compressed sensing kalman filter approaches are given for BPSK data. Finally, the critical issue of smart grid security is addressed, and a possible approach for achieving this is presented using FPGA-based fuzzy logic intrusion detection.

\section{Acknowledgments}

This work is funded by the National Science Foundation through Grants (ECCS-0901420), (ECCS-0821658), and (ECCS-0622125) and the Office of Naval Research through two Contracts (N00014-07-1-0529 and N00014-11-1-0006).

\section{References}

[1] J. Mitola III and G. Q. Maguire Jr., "Cognitive radio: making software radios more personal," IEEE Personal Communications, vol. 6, no. 4, pp. 13-18, 1999.

[2] S. Haykin, "Cognitive radio: brain-empowered wireless communications," IEEE Journal on Selected Areas in Communications, vol. 23, no. 2, pp. 201-220, 2005.

[3] G. Ganesan, Y. Li, B. Bing, and S. Li, "Spatiotemporal sensing in cognitive radio networks," IEEE Journal on Selected Areas in Communications, vol. 26, no. 1, pp. 5-12, 2008.

[4] J. Bazerque and G. Giannakis, "Distributed spectrum sensing for cognitive radio networks by exploiting sparsity," IEEE Transactions on Signal Processing, vol. 58, no. 3, pp. 1847-1862, 2010.

[5] C. Cordeiro, K. Challapali, D. Birru et al., "IEEE 802.22: an introduction to the first wireless standard based on cognitive radios," Journal of Communications, vol. 1, no. 1, pp. 38-47, 2006.

[6] C. Cordeiro, K. Challapali, D. Birru, and N. Sai Shankar, "IEEE 802.22: the first worldwide wireless standard based on cognitive radios," in Proceedings of the 1st IEEE International Symposium on New Frontiers in Dynamic Spectrum Access Networks (DySPAN '05), pp. 328-337, Baltimore, Md, USA, November 2005.
[7] C. Cordeiro, K. Challapali, and M. Ghosh, "Cognitive PHY and MAC layers for dynamic spectrum access and sharing of TV bands," in Proceedings of the 1st International Workshop on Technology and Policy for Accessing Spectrum, vol. 222, p. 3, ACM, New York, NY, USA, 2006.

[8] C. Stevenson, G. Chouinard, Z. Lei, W. Hu, S. Shellhammer, and W. Caldwell, "IEEE 802.22: the first cognitive radio wireless regional area network standard," IEEE Communications Magazine, vol. 47, no. 1, pp. 130-138, 2009.

[9] Z. Jiang, "Computational intelligence techniques for a smart electric grid of the future," in Proceedings of the 6th International Symposium on Neural Networks on Advances in Neural Networks (ISNN '09), pp. 1191-1201, 2009.

[10] Z. Wang, A. Scaglione, and R. J. Thomas, "Compressing electrical power grids," in Proceedings of the 1st IEEE International Conference on Smart Grid Communications (SmartGridComm '10), pp. 13-18, 2010.

[11] A. Mohsenian-Rad, V. Wong, J. Jatskevich, and R. Schober, "Optimal and autonomous incentive-based energy consumption scheduling algorithm for smart grid," in Proceedings of the Innovative Smart Grid Technologies (ISGT '10), pp. 1-6, Citeseer, Gaithersburg, Md, USA, January 2010.

[12] S. Caron and G. Kesidis, "Incentive-based energy consumption scheduling algorithms for the smart grid," in Proceedings of the 1st IEEE International Conference on Smart Grid Communications, pp. 391-396, Gaithersburg, Md, USA, October 2010.

[13] A. L. Dimeas and N. D. Hatziargyriou, "Operation of a multiagent system for microgrid control," IEEE Transactions on Power Systems, vol. 20, no. 3, pp. 1447-1455, 2005.

[14] S. Hatami and M. Pedram, "Minimizing the electricity bill of cooperative users under a Quasi-Dynamic Pricing Model," in Proceedings of the 1st IEEE International Conference on Smart Grid Communications (SmartGridComm '10), pp. 421-426, IEEE, 2010.

[15] P. Samadi, A. Mohsenian-Rad, R. Schober, V. Wong, and J. Jatskevich, "Optimal real-time pricing algorithm based on utility maximization for smart grid," in Proceedings of the IEEE International Conference on Smart Grid (SmartGridComm '10), Gaithersburg, Mass, USA, October 2010.

[16] J. F. Hauer, N. B. Bhatt, K. Shah, and S. Kolluri, "Performance of "WAMS East" in providing dynamic information for the North East blackout of August 14, 2003," in Proceedings of the IEEE Power Engineering Society General Meeting, pp. 16851690, IEEE, Denver, Colo, USA, June 2004.

[17] D. Divan, G. A. Luckjiff, W. E. Brumsickle, J. Freeborg, and A. Bhadkamkar, "A grid information resource for nationwide real-time power monitoring," IEEE Transactions on Industry Applications, vol. 40, no. 2, pp. 699-705, 2004.

[18] B. Qiu, L. Chen, V. Centeno, X. Dong, and Y. Liu, "Internet based frequency monitoring network (FNET)," in Proceedings of the IEEE Power Engineering Society Winter Meeting, vol. 3, pp. 1166-1171, IEEE, 2002.

[19] A. G. Phadke, "Synchronized phasor measurements in power systems," IEEE Computer Applications in Power, vol. 6, no. 2, pp. 10-15, 1993.

[20] Z. Zhong, C. Xu, B. J. Billian et al., "Power system frequency monitoring network (FNET) implementation," IEEE Transactions on Power Systems, vol. 20, no. 4, pp. 1914-1921, 2005.

[21] S. Tsai, Z. Zhong, J. Zuo, and Y. Liu, "Analysis of widearea frequency measurement of bulk power systems," in Proceedings of the IEEE Power Engineering Society General Meeting, Montreal, Canada, June 2006. 
[22] C. Chang, A. Liu, and C. Huang, "Oscillatory stability analysis using real-time measured data," IEEE Transactions on Power Systems, vol. 8, no. 3, pp. 823-829, 2002.

[23] C. Chunling, X. Tongyu, P. Zailin, and Y. Ye, "Power quality disturbances classification based on multi-class classification SVM," in Proceedings of the 2nd International Conference on Power Electronics and Intelligent Transportation System (PEITS '09), vol. 1, pp. 290-294, IEEE, 2009.

[24] P. Gao and W. Wu, "Power quality disturbances classification using wavelet and support vector machines," in Proceedings of the 6st International Conference on Intelligent Systems Design and Applications, (ISDA '06), pp. 201-206, October 2006.

[25] A. M. Gaouda, S. H. Kanoun, M. M. A. Salama, and A. Y. Chikhani, "Pattern recognition applications for power system disturbance classification," IEEE Transactions on Power Delivery, vol. 17, no. 3, pp. 677-683, 2002.

[26] F. Melgani and Y. Bazi, "Classification of electrocardiogram signals with support vector machines and particle swarm optimization," IEEE Transactions on Information Technology in Biomedicine, vol. 12, no. 5, pp. 667-677, 2008.

[27] I. Guler and E. D. Ubeyli, "Multiclass support vector machines for EEG-signals classification," IEEE Transactions on Information Technology in Biomedicine, vol. 11, no. 2, pp. 117-126, 2007.

[28] C. Cortes and V. Vapnik, "Support-vector networks," Machine Learning, vol. 20, no. 3, pp. 273-297, 1995.

[29] N. I. of Standards and Technologies, "Guidelines for grid security, vol 1,” Tech. Rep., 2010, http://csrc.nist.gov/publications/ PubsNISTIRs.html.

[30] M. Pazos-Revilla and A. Siraj, "An experimental model of an fpga-based intrusion detection systems," in Proceedings of the 26th International Conference on Computers and Their Applications, 2011.

[31] R. C. Qiu, Z. Chen, N. Guo et al., "Towards a realtime cognitive radio network testbed: architecture, hardware platform, and application to smart grid," in Proceedings of the 5th IEEE Workshop on Networking Technologies for SoftwareDefined Radio and White Space, June 2010.

[32] Z. Chen, N. Guo, and R. C. Qiu, "Building A cognitive radio network testbed," in Proceedings of the IEEE Southeastcon, Nashville, Tenn, USA, March 2011.

[33] R. C. Qiu, “Cognitive radio network testbed," Funded Research Proposal for Defense University Research Instrumentation Program (DURIP), August 2009, http://www .defense.gov/news/Fiscal 2010 DURIP Winners List.pdf.

[34] R. C. Qiu, "Cognitive radio and smart grid," Invited Presentation at IEEE Chapter, February 2010, http://iweb.tntech .edu/rqiu.

[35] R. C. Qiu, “Cogntiive radio institute," Funded research proposal for 2010 Defense Earmark, 2010, http://www.opensecrets.org/politicians/earmarks.php?cid=N00003126.

[36] R. C. Qiu, "Smart grid research at TTU," Presented at Argonne National Laboratory, February 2010, http://iweb.tntech.edu/ rqiu/publications.htm.

[37] R. Qiu, Z. Hu, G. Zheng, Z. Chen, and N. Guo, "Cognitive radio network for the Smart Grid: experimental system architecture, control algorithms, security, and microgrid testbed," IEEE Transactions on Smart Grid. In press.

[38] R. C. Qiu, M. C. Wicks, Z. Hu, L. Li, and S. J. Hou, "Wireless tomography(part1): a novel approach to remote sensing," in Proceedings of the 5th International Waveform Diversity and Design Conference, Niagara Falls, Canada, August 2010.
[39] M. Amin and B. Wollenberg, "Toward a smart grid: power delivery for the 21st century," IEEE Power and Energy Magazine, vol. 3, no. 5, pp. 34-41, 2005.

[40] J. Cupp and M. Beehler, "Implementing smart grid communications," 2008.

[41] A. Ghassemi, S. Bavarian, and L. Lampe, "Cognitive radio for smart grid communications," in Proceedings of the 1st IEEE International Conference on Smart Grid Communications (SmartGridComm '10), pp. 297-302, IEEE, Gaithersburg, Md, USA, 2010.

[42] N. Ghasemi and S. M. Hosseini, "Comparison of smart grid with cognitive radio: solutions to spectrum scarcity," in Proceedings of the 12th International Conference on Advanced Communication Technology (ICACT '10), vol. 1, pp. 898-903, February 2010.

[43] J. Lee and M. Verleysen, Nonlinear Dimensionality Reduction, Springer, London, UK, 2007.

[44] I. T. Jolliffe, Principal Component Analysis, Springer, London, UK, 2002.

[45] B. Schölkopf, A. Smola, and K. R. Müller, "Nonlinear component analysis as a kernel eigenvalue problem," Neural Computation, vol. 10, no. 5, pp. 1299-1319, 1998.

[46] K. Q. Weinberger and L. K. Saul, "Unsupervised learning of image manifolds by semidefinite programming," International Journal of Computer Vision, vol. 70, no. 1, pp. 77-90, 2006.

[47] K. Weinberger, B. Packer, and L. Saul, "Nonlinear dimensionality reduction by semidefinite programming and kernel matrix factorization," in Proceedings of the 10th International Workshop on Artificial Intelligence and Statistics, pp. 381-388, 2005.

[48] V. Vapnik, The Nature of Statistical Learning Theory, Springer, London, UK, 2000.

[49] V. Vapnik, Statistical Learning Theory, Wiley, New York, NY, USA, 1998.

[50] V. Vapnik, S. Golowich, and A. Smola, "Support vector method for function approximation, regression estimation, and signal processing," in Advances in Neural Information Processing Systems, M. Mozer, M. Jordan, and T. Petsche, Eds., pp. 281-287, MIT Press, Cambridge, Mass, USA, 1997.

[51] C. J. C. Burges, "A tutorial on support vector machines for pattern recognition," Data Mining and Knowledge Discovery, vol. 2, no. 2, pp. 121-167, 1998.

[52] A. J. Smola and B. Schölkopf, "A tutorial on support vector regression," Statistics and Computing, vol. 14, no. 3, pp. 199222, 2004.

[53] N. Cristianini and J. Shawe-Taylor, An Introduction to Support Vector Machines: and Other Kernel-Based Learning Methods, Cambridge University Press, Cambridge, UK, 2000.

[54] Z. Chen and R. C. Qiu, "Prediction of channel state for cognitive radio using higher-order hidden Markov model," in Proceedings of the IEEE Southeast Conference, pp. 276-282, March 2010.

[55] J. Sturm, “The advanced optimization laboratory at McMaster university, Canada. SeDuMi version 1.1 R3," 2006.

[56] S. Canu, Y. Grandvalet, V. Guigue, and A. Rakotomamonjy, Svm and Kernel Methods Matlab Toolbox, Perception Systmes et Information, INSA de Rouen, Rouen, France, 2005.

[57] D. Fradkin and I. Muchnik, "Support vector machines for classification," Discrete Methods in Epidemiology, vol. 70, pp. 13-20, 2006.

[58] K. Bennett and C. Campbell, "Support vector machines: hype or hallelujah?” ACM SIGKDD Explorations Newsletter, vol. 2, no. 2, pp. 1-13, 2000. 
[59] D. L. Donoho, "Compressed sensing," IEEE Transactions on Information Theory, vol. 52, no. 4, pp. 1289-1306, 2006.

[60] Y. Tsaig and D. L. Donoho, "Extensions of compressed sensing," Signal Processing, vol. 86, no. 3, pp. 549-571, 2006.

[61] E. Candès, "The restricted isometry property and its implications for compressed sensing," Comptes Rendus Mathematique, vol. 346, no. 9-10, pp. 589-592, 2008.

[62] E. Candès, J. Romberg, and T. Tao, "Robust uncertainty principles: exact signal reconstruction from highly incomplete frequency information," IEEE Transactions on Information Theory, vol. 52, no. 2, pp. 489-509, 2006.

[63] Z. Tian and G. B. Giannakis, "Compressed sensing for wideband cognitive radios," in Proceedings of IEEE International Conference on Acoustics, Speech and Signal Processing (ICASSP '07), vol. 4, pp. 1357-1360, 2007.

[64] L. Husheng, M. Rukun, L. Lifeng, and R. Qiu, "Compressed meter reading for delay-sensitive and secure load report in smart grid," in Proceedings of the 1st IEEE International Conference on Smart Grid Communications (SmartGridComm '10), 2010.

[65] S. Ji, Y. Xue, and L. Carin, "Bayesian compressive sensing," IEEE Transactions on Signal Processing, vol. 56, no. 6, pp. 2346 2356, 2008.

[66] A. Carmi, P. Gurfil, and D. Kanevsky, "Methods for sparse signal recovery using Kalman filtering with embedded pseudomeasurement norms and quasi-norms," IEEE Transactions on Signal Processing, vol. 58, no. 4, pp. 2405-2409, 2010.

[67] S. S. Chen, D. L. Donoho, and M. A. Saunders, "Atomic decomposition by basis pursuit," SIAM Review, vol. 43, no. 1, pp. 129-159, 2001.

[68] S. G. Mallat and Z. Zhang, "Matching pursuits with timefrequency dictionaries," IEEE Transactions on Signal Processing, vol. 41, no. 12, pp. 3397-3415, 1993.

[69] J. A. Tropp and A. C. Gilbert, "Signal recovery from random measurements via orthogonal matching pursuit," IEEE Transactions on Information Theory, vol. 53, no. 12, pp. 4655-4666, 2007.

[70] R. Meinhold and N. Singpurwalla, "Understanding the Kalman filter," American Statistician, vol. 37, no. 2, pp. 123 127, 1983.

[71] R. Kalman et al., "A new approach to linear filtering and prediction problems," Journal of Basic Engineering, vol. 82, no. 1, pp. 35-45, 1960.

[72] S. Haykin, Adaptive Filter Theory, Pearson Education, Dorling Kindersley ,India, 2008.

[73] E. Wan and R. van der Merwe, "The unscented Kalman filter for nonlinear estimation," in Proceedings of the Adaptive Systems for Signal Processing, Communications, and Control Symposium (AS-SPCC '00), pp. 153-158, IEEE, 2000.

[74] G. Evensen, "The ensemble Kalman filter: theoretical formulation and practical implementation," Ocean Dynamics, vol. 53, no. 4, pp. 343-367, 2003.

[75] L. Ma, K. Wu, and L. Zhu, "Fire smoke detection in video images using Kalman filter and Gaussian mixture color model," in Proceedings of the International Conference on Artificial Intelligence and Computational Intelligence (AICI '10), vol. 1, pp. 484-487, IEEE, Sanya, China, 2010.

[76] L. Ljung, "Asymptotic behavior of the extended Kalman filter as a parameter estimator for linear systems," IEEE Transactions on Automatic Control, vol. 24, no. 1, pp. 36-50, 2002.

[77] R. van der Merwe and E. Wan, "The square-root unscented Kalman filter for state and parameter-estimation," in Proceedings of the IEEE International Conference on Acoustics, Speech, and Signal Processing (ICASSP '01), vol. 6, pp. 3461-3464, IEEE, Salt Lake City, Utah, USA, 2001.

[78] A. Lakhzouri, E. Lohan, R. Hamila, and M. Renfors, "Extended Kalman filter channel estimation for line-of-sight detection in WCDMA mobile positioning," EURASIP Journal on Applied Signal Processing, vol. 2003, pp. 1268-1278, 2003.

[79] D. Kanevsky, A. Carmi, L. Horesh, P. Gurfil, B. Ramabhadran, and T. Sainath, "Kalman filtering for compressed sensing," in Proceedings of the 13th Conference on Information Fusion (FUSION '10), pp. 1-8, Edinburgh, UK, July 2010.

[80] S. J. Julier and J. J. LaViola, "On Kalman filtering with nonlinear equality constraints," IEEE Transactions on Signal Processing, vol. 55, no. 6, pp. 2774-2784, 2007.

[81] M. E. Tipping, "Sparse bayesian learning and the relevance vector machine," Journal of Machine Learning Research, vol. 1, no. 3, pp. 211-244, 2001.

[82] M. Pazos-Revilla, Fpga based fuzzy intrusion detection system for network security, M.S. thesis, Tennessee Technological University, Cookeville, Tenn, USA, 2010.

[83] W. Sanders, "Tcip: trustworthy cyber infrastructure for the power grid,” Tech. Rep., Information Trust Institute, University of Illinois at Urbana-Champaign, 2011.

[84] R. Berthier, W. Sanders, and H. Khurana, "Intrusion detection for advanced metering infrastructures: requirements and architectural directions," in Proceedings of the 1st IEEE International Conference on Smart Grid Communications (SmartGridComm '10), pp. 350-355, IEEE, Gaithersburg, Md, USA, October 2010. 

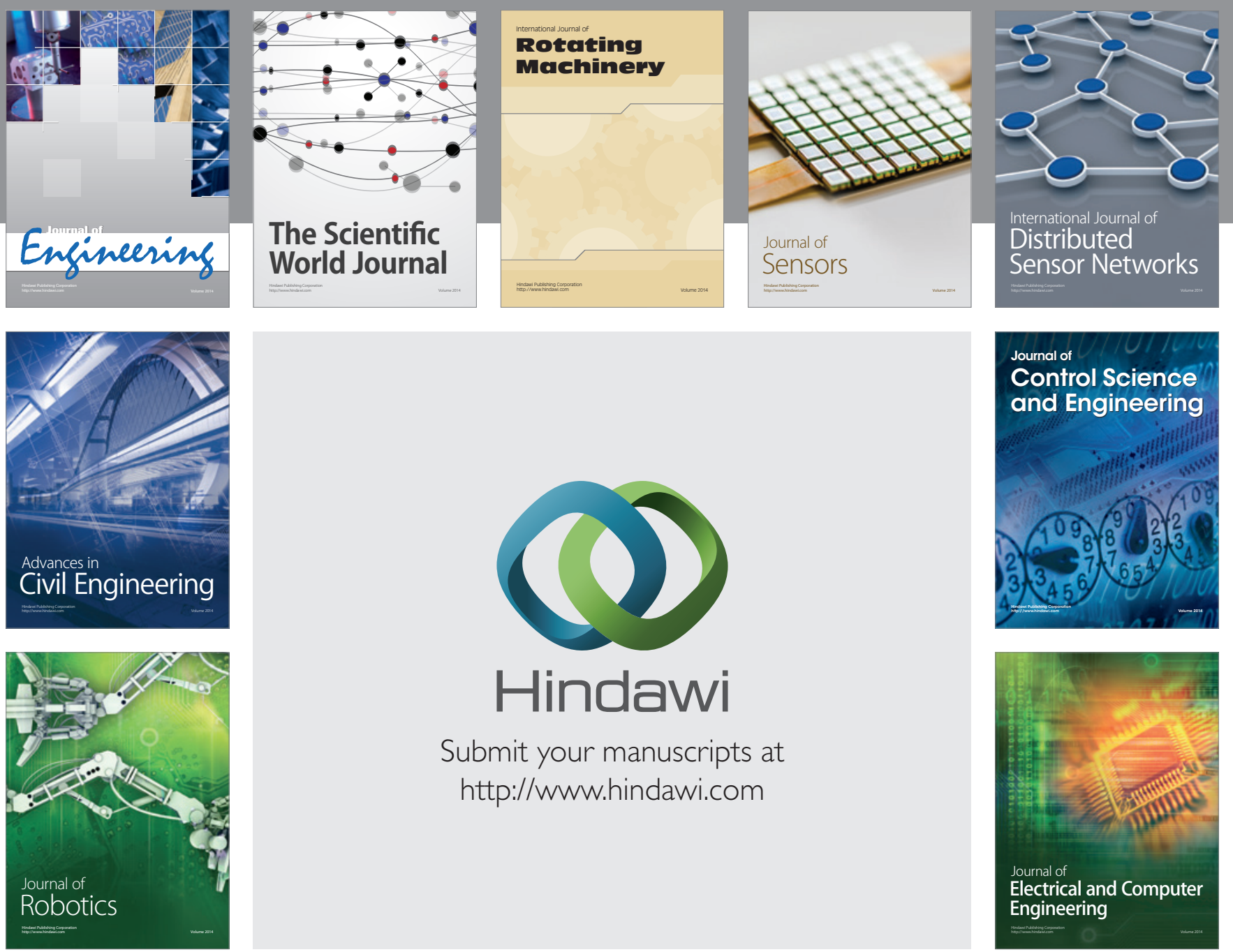

Submit your manuscripts at

http://www.hindawi.com
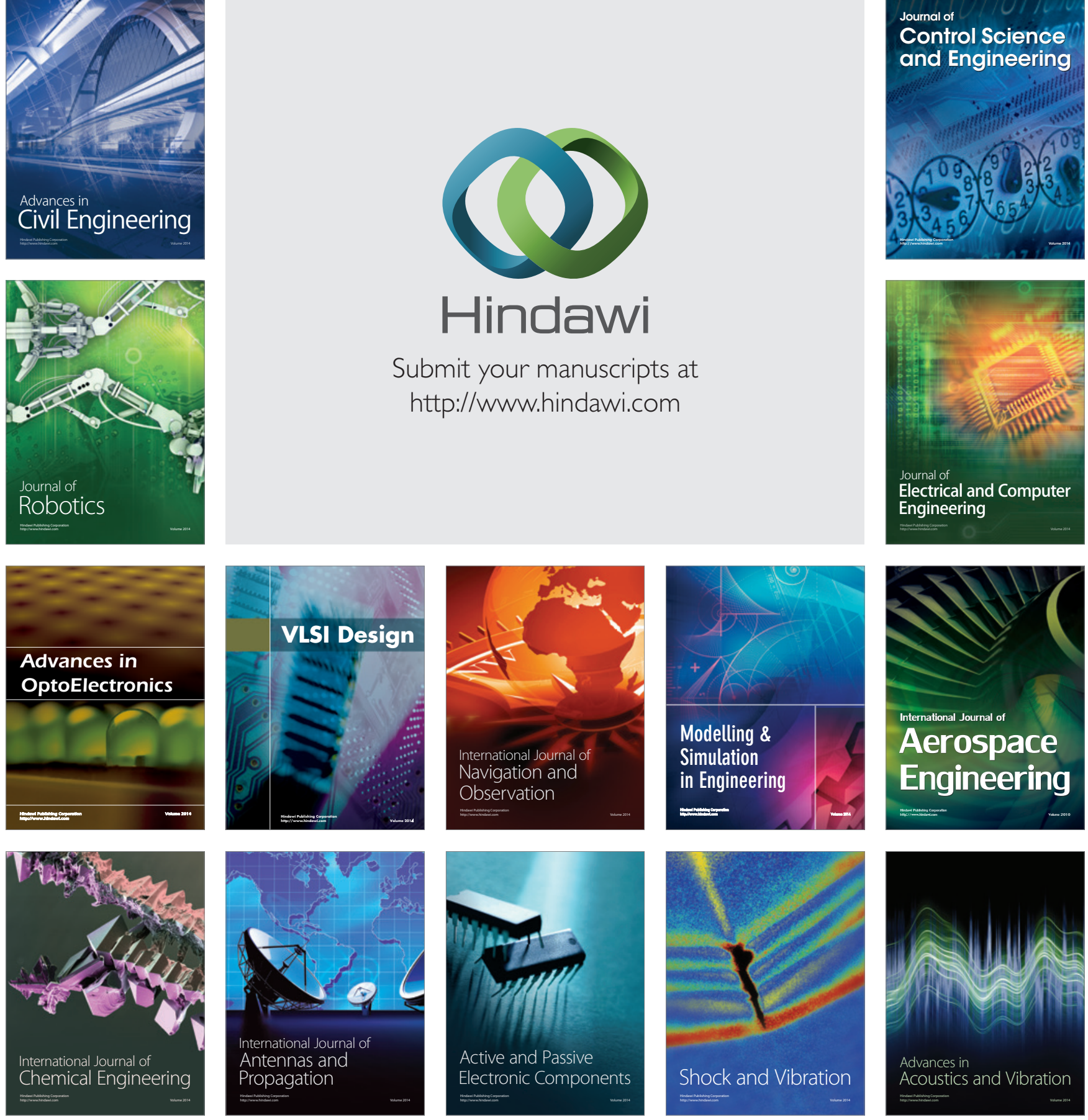